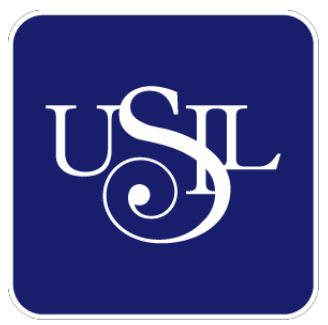

UNIVERSIDAD

SAN IGNACIO

DE LOYOLA

FACULTAD DE INGENIERÍA

Carrera de Ingeniería Industrial y Comercial

\title{
MEJORA DE LA GESTIÓN DE ADMINISTRACIÓN DE REGISTROS PARA REDUCIR LOS RECURSOS Y TIEMPO DE ATENCIÓN AL CLIENTE
}

Tesis para optar el Título Profesional de Ingeniero

Industrial y Comercial

MELISSA VALERIA AGURTO MARZULLO

(0000-0001-5764-1552)

Asesor:

Mg. Tulio Elías Florián Castillo

(0000-0002-7748-2699)

Lima - Perú

2021 


\title{
JURADO DE LA SUSTENTACIÓN ORAL
}

\author{
Presidente
}

Jurado 1

\section{Jurado 2}

Entregado el:

$\mid 20 \ldots$

Aprobado por:

(Nombres y Apellidos completos del Bachiller)

Graduando

(Nombres y Apellidos completos del Asesor) Asesor de Tesis 


\section{EPÍGRAFE}

"La eficiencia es hacer cosas bien; la efectividad es hacer las cosas correctas"

\section{Peter F. Drucker}




\section{UNIVERSIDAD SAN IGNACIO DE LOYOLA FACULTAD DE INGENIERÍA DECLARACIÓN DE AUTENTICIDAD}

Yo, Melissa Valeria Agurto Marzullo, identificado con DNI № 72077736, Bachiller del Programa Académico de la Carrera de Ingeniería Industrial y Comercial de la Facultad de Ingeniería de la Universidad San Ignacio de Loyola, presento mi tesis titulada:

"Mejora de la gestión de Administración de Registros para reducir los recursos y tiempo de atención al cliente".

Declaro en honor a la verdad, que el trabajo de tesis es de mi autoría; que los datos, los resultados y su análisis e interpretación, constituyen mi aporte. Todas las referencias han sido debidamente consultadas y reconocidas en la investigación.

En tal sentido, asumo la responsabilidad que corresponda ante cualquier falsedad $u$ ocultamiento de la información aportada. Por todas las afirmaciones ratifico lo expresado, a través de mi firma correspondiente.

Lima, 15 de enero de 2020

Melissa Valeria Agurto Marzullo

DNI: 72077736 
ÍNDICE DE CONTENIDOS

$\begin{array}{ll}\text { INTRODUCCIÓN } & 17\end{array}$

$\begin{array}{ll}\text { PROBLEMA DE INVESTIGACIÓN } & 18\end{array}$

$\begin{array}{ll}\text { Identificación del problema } & 18\end{array}$

$\begin{array}{ll}\text { Formulación del problema } & 23\end{array}$

MARCO REFERENCIAL 24

$\begin{array}{ll}\text { Antecedentes } & 24\end{array}$

Estado del arte $\quad 26$

$\begin{array}{ll}\text { Marco Teórico } & 27\end{array}$

$\begin{array}{ll}\text { OBJETIVOS } & 37\end{array}$

$\begin{array}{ll}\text { Objetivo General } & 37\end{array}$

$\begin{array}{ll}\text { Objetivos Específicos } & 37\end{array}$

$\begin{array}{ll}\text { JUSTIFICACIÓN } & 38\end{array}$

$\begin{array}{ll}\text { Teórica } & 38\end{array}$

$\begin{array}{ll}\text { Práctica } & 38\end{array}$

$\begin{array}{ll}\text { Social } & 39\end{array}$

$\begin{array}{ll}\text { HIPÓTESIS } & 40\end{array}$

Hipótesis General $\quad 40$

$\begin{array}{ll}\text { Hipótesis Específicas } & 40\end{array}$

MATRIZ DE CONSISTENCIA 41

MARCO METODOLÓGICO 44

$\begin{array}{lr}\text { Paradigma } & 44\end{array}$

$\begin{array}{ll}\text { Enfoque } & 44\end{array}$

$\begin{array}{ll}\text { Método } & 44\end{array}$

$\begin{array}{ll}\text { VARIABLES } & 45\end{array}$ 
$\begin{array}{ll}\text { Independiente } & 45\end{array}$

$\begin{array}{ll}\text { Dependiente } & 45\end{array}$

$\begin{array}{ll}\text { POBLACIÓN Y MUESTRA } & 46\end{array}$

$\begin{array}{ll}\text { Población } & 46\end{array}$

$\begin{array}{ll}\text { Muestra } & 49\end{array}$

UNIDAD DE ANÁLISIS

$\begin{array}{ll}\text { Técnica } & 51\end{array}$

Instrumento

PROCEDIMIENTO Y MÉTODO DE ANÁLISIS 52

$\begin{array}{ll}\text { Procedimiento } & 52\end{array}$

Método de análisis $\quad 88$

$\begin{array}{lr}\text { RESULTADOS } & 97\end{array}$

$\begin{array}{lr}\text { CONCLUSIONES } & 129\end{array}$

$\begin{array}{lr}\text { RECOMENDACIONES } & 130\end{array}$

$\begin{array}{ll}\text { REFERENCIAS } & 132\end{array}$

$\begin{array}{ll}\text { ANEXOS } & 134\end{array}$ 


\section{ÍNDICE DE TABLAS}

Tablas

Tabla 1: Toma de tiempos por proceso. 19

Tabla 2: Formulario General del estudio de tiempos. 33

Tabla 3: Simbología diagrama de flujo. $\quad 34$

Tabla 4: Matriz de Consistencia. 43

Tabla 5: Población por área. 48

Tabla 6: Áreas de encuesta. 51

Tabla 7: Estrategia de liberación de documentos SAP. 54

Tabla 8: Procesos asignados por registrador. 66

Tabla 9: Registrador asignado a locales. $\quad 66$

Tabla 10: Resumen de la toma de tiempos por proceso. 68

Tabla 11: Toma de tiempos del proceso de solicitud de cotización. 69

Tabla 12: Toma de tiempos del proceso de solicitud de pedido. 71

Tabla 13: Toma de tiempos del proceso de pedido descentralizado. 73

Tabla 14: Toma de tiempos del proceso de pedido centralizado con acuerdo de compra. $\quad 75$

Tabla 15: Toma de tiempos del proceso de contabilizaciones descentralizadas. $\quad 77$

Tabla 16: Toma de tiempos del proceso de contabilizaciones centralizadas. $\quad 79$

Tabla 17: Toma de tiempos del proceso de creación de códigos de materiales. 81

Tabla 18: Toma de tiempos del proceso de creación de código de acreedores. 83

Tabla 19: Toma de tiempos del proceso de reserva de materiales. 84

Tabla 20: Toma de tiempos del proceso de anticipos. 86

Tabla 21: Personal de las áreas independientes a encuestar. 88

Tabla 22: Resultados pregunta 1 - Encuesta. $\quad 89$

Tabla 23: Resultados pregunta 2 - Encuesta. 90

Tabla 24: Resultados pregunta 3 - Encuesta. 91

Tabla 25: Resultados pregunta 4 - Encuesta. 92

Tabla 26: Resultados pregunta 5 - Encuesta. 93

Tabla 27: Resultados pregunta 6 - Encuesta. 94

Tabla 28: Resultados pregunta 7 - Encuesta. 95 
Tabla 29: Resumen del tiempo inicial por proceso. 109

Tabla 30: Resumen del tiempo final por proceso 110

Tabla 31: Cantidad de FTE para los requerimientos atendidos por Administración de Registros. 112

Tabla 32: Cantidad de FTE para los requerimientos atendidos por áreas independientes. 112

Tabla 33: Cantidad total de FTE por proceso. 114

Tabla 34: Identificación de cantidad de documentos de compra - Octubre 2019. 115

Tabla 35: Plan de capacitación: Módulo MM SAP. 116

Tabla 36: Costo total de licencias SAP de las 127

Tabla 37: Resumen de costos por licencia asignadas a las áreas independientes. 


\section{ÍNDICE DE GRÁFICOS}

Gráficos

Gráfico 1: Diagrama de Ishikawa 20

Gráfico 2: Porcentaje de distribución de cargas en el sistema SAP 2019.

Gráfico 3: Porcentaje de distribución de cargas de otras áreas independientes en el sistema SAP 2019.

Gráfico 4: Diagrama de Ishikawa (causa - efecto) 29

Gráfico 5: Organigrama del área de administración de registros 47

Gráfico 6: Porcentaje de la población por área. 48

Gráfico 7: Diagrama de flujo - Solicitud de cotización. 56

Gráfico 8: Diagrama de flujo - Solicitud de pedido. 57

Gráfico 9: Diagrama de flujo - Pedido descentralizado. 58

Gráfico 10: Diagrama de flujo - Pedido centralizado con acuerdo de compra. $\quad 59$

Gráfico 11: Diagrama de flujo - Contabilización descentralizada. 60

Gráfico 12: Diagrama de flujo - Contabilizaciones centralizadas. 61

Gráfico 13: Diagrama de flujo - Creación de código de materiales. 62

Gráfico 14: Diagrama de flujo - Creación de código de acreedores. 63

Gráfico 15: Diagrama de flujo - Reserva de materiales. 64

Gráfico 16: Diagrama de flujo - Anticipos. 65

Gráfico 17: Resumen de toma de tiempos por registrador 70

Gráfico 18: Resumen de toma de tiempos por registrador 72

Gráfico 19: Ranking de toma de tiempos por registrador 74

Gráfico 20: Resumen de toma de tiempos por registrador para el proceso de pedido centralizado con acuerdo de compra. $\quad 76$

Gráfico 21: Resumen de toma de tiempos por registrador para el proceso de contabilizaciones descentralizadas.

Gráfico 22: Resumen de toma de tiempos por registrador para el proceso de contabilizaciones centralizadas.

Gráfico 23: Resumen de toma de tiempos por registrador para el proceso de creación de código de materiales.

Gráfico 24: Resumen de toma de tiempos por registrador para el proceso de creación de reserva de materiales. 
Gráfico 25: Resumen de toma de tiempos por registrador para el proceso de anticipos.

Gráfico 26: Resultados pregunta 1 - Encuesta. $\quad 89$

Gráfico 27: Resultados pregunta 2 - Encuesta. 90

Gráfico 28: Resultados pregunta 3 - Encuesta. $\quad 91$

Gráfico 29: Resultados pregunta 4 - Encuesta. 92

Gráfico 30: Resultados pregunta 5 - Encuesta. 94

Gráfico 31: Resultados pregunta 6 - Encuesta. 95

Gráfico 32: Resultados pregunta 7 - Encuesta. 96

Gráfico 33: Tiempo promedio inicial vs final para el proceso de solicitud de cotización. 98

Gráfico 34: Tiempo promedio inicial vs final para el proceso de solicitud de pedido.

Gráfico 35: Tiempo promedio inicial vs final para el proceso de pedido descentralizado

Gráfico 36: Tiempo promedio inicial vs final para el proceso de pedido centralizado con acuerdo de compra.

Gráfico 37: Tiempo promedio inicial vs final para el proceso de contabilizaciones descentralizadas.

Gráfico 38: Tiempo promedio inicial vs final para el proceso de contabilizaciones centralizadas.

Gráfico 39: Tiempo promedio inicial vs final para el proceso de creación de código de materiales.

Gráfico 40: Tiempo promedio inicial vs final para el proceso de creación de código de acreedor.

Gráfico 41: Tiempo promedio inicial vs final para el proceso de reserva de materiales.

Gráfico 42: Tiempo promedio inicial vs final para el proceso de anticipos. 107

Gráfico 43: Días pico por mes - FTE máximo. 113

Gráfico 44: Diagrama de Pareto de documentos de compra - Octubre $2019 \quad 115$

Gráfico 45: Diagrama de flujo 2 - Solicitud de cotización. 118

Gráfico 46: Diagrama de flujo 2 - Solicitud de pedido 119

Gráfico 47: Diagrama de flujo 2 - Pedido descentralizado. 120 
Gráfico 48: Diagrama de flujo 2 - Pedido centralizado con acuerdo de compra. 121

Gráfico 49: Diagrama de flujo 2 - Contabilizaciones descentralizadas. 122

Gráfico 50: Diagrama de flujo 2 - Contabilizaciones centralizadas. 123

Gráfico 51: Diagrama de flujo 2 - Reserva de materiales. 124

Gráfico 52: Diagrama de flujo 2 - Anticipos. 125 


\section{ÍNDICE DE ANEXOS}

Anexos

Anexo 1: Ejemplo base de datos de documentos de compra SAP. 134

Anexo 2. Formato de Solicitud de Cotización 135

Anexo 3. Formato de Solicitud de Pedido 136

Anexo 4. Formato de Pedido Descentralizado 137

Anexo 5. Formato de Solicitud Anticipo de Personal 138

Anexo 6. Formato de Solicitud de Anticipo Proveedor 139

Anexo 7. Formato de Reserva de Materiales $\quad 140$

Anexo 8: Encuesta de evaluación del área de Administración de Registros. $\quad 141$

Anexo 9: Reporte de Documentos de Compra - SAP octubre 2019. 


\section{DEDICATORIA}

Este presente trabajo es dedicado a mis queridos padres por darme la fuerza y apoyo incondicional en cada peldaño de la vida. 


\section{AGRADECIMIENTO}

Agradezco a la Universidad San Ignacio de Loyola por brindarme los conocimientos necesarios para mi formación académica y profesional, y sobre todo a Dios por ser mi fortaleza ante cualquier obstáculo. 


\section{RESUMEN}

En esta investigación se ha seleccionado el área de Administración de Registros de un operador logístico cuyo objetivo del área es brindar soporte a las áreas del core del negocio, a través de la creación de documentos de compra en el sistema SAP, para la adquisición de un bien o servicio. Sin embargo, esta área presenta una inadecuada gestión y asignación de recursos que estaría perjudicando la continuidad de las áreas usuarias por demoras en el tiempo de atención y generando costos adicionales a la empresa.

Asimismo, como parte de la investigación se realizaron entrevistas a las áreas usuarias (clientes internos) corroborando la insatisfacción en cuanto a la efectividad del área seleccionada.

En tal sentido, el desarrollo de esta tesis tiene como propósito la optimización del tiempo de atención y recursos de Administración de Registros aplicando técnicas y herramientas de eficiencia y mejora continua de la ingeniería: Diagrama de Ishikawa (causa - efecto), diagrama de flujo y medición del trabajo (estudio de tiempos). En adición, se realizará el cálculo de la cantidad del personal que se requiere para cubrir la demanda mensual, mediante el cálculo de un equivalente a tiempo completo (FTE).

Producto del análisis y resultados de las técnicas aplicadas se presentará la comparación de la situación actual versus la propuesta como estrategia de mejora.

\section{Palabras clave: Efectividad, optimización del tiempo, asignación de recursos, mejora continua, medición del trabajo, estudio de tiempos.}




\begin{abstract}
In this research, the Logistics Management area of a logistics operator has been selected, whose objective is to provide support to the business core areas, through the creation of purchase documents in the SAP system, for the acquisition of a good or service However, this area presents an inadequate management and allocation of resources that will damage the continuity of the user areas due to delays in the time of attention and generating additional costs in the company.
\end{abstract}

Likewise, as part of the investigation, interviews are conducted with the user areas (internal clients) that corroborate the dissatisfaction regarding the strength of the selected area.

In this sense, the development of this thesis has the purpose of optimizing the time, attention and resources of records management, technical application, efficiency and continuous improvement of engineering: Ishikawa diagram (cause - effect), flow chart and work measurement. In the case that it is a calculation of time equivalent to a full time (FTE).

Product analysis and results of the applied techniques present the comparison of the current situation with the proposal as an improvement strategy.

Key words: Effectiveness, optimization of time, allocation of resources, continuous improvement, measurement of work, study of times. 